\title{
Measurements of Superattenuator seismic isolation by Virgo interferometer
}

F. Acernese ${ }^{g, i}$, F. Antonucci ${ }^{\circ}$, S. Aoudia ${ }^{\mathrm{y}}$, K.G. Arun ${ }^{\mathrm{t}}$, P. Astone ${ }^{\mathrm{o}}$, G. Ballardin ${ }^{\mathrm{b}}, \mathrm{F}$. Barone $^{\mathrm{g}, \mathrm{i}}$, M. Barsuglia $^{\mathrm{a}}$, Th.S. Bauer ${ }^{\mathrm{w}}$, M.G. Beker ${ }^{\mathrm{w}}$, S. Bigotta ${ }^{\mathrm{l}, \mathrm{m}}$, S. Birindelli ${ }^{\mathrm{y}}$, M. Bitossi ${ }^{\mathrm{l}}$, M.A. Bizouard ${ }^{\mathrm{t}}$, M. Blom ${ }^{\mathrm{w}}$, C. Boccara $^{\mathrm{c}}$, F. Bondu ${ }^{\mathrm{z}}$, L. Bonelli ${ }^{\mathrm{l}, \mathrm{m}}$, L. Bosi $^{\mathrm{j}}, \mathrm{S}$. Braccini ${ }^{1, *}$, C. Bradaschia ${ }^{\mathrm{l}}$, A. Brillet $^{\mathrm{y}}, \mathrm{V}$. Brisson ${ }^{\mathrm{t}}$, R. Budzyński ${ }^{\text {af }}$, T. Bulik ${ }^{\text {ag,ah }}$, H.J. Bulten ${ }^{\mathrm{w}, \mathrm{x}}$, D. Buskulic ${ }^{\mathrm{u}}$, G. Cagnoli $^{\mathrm{d}}$, E. Calloni ${ }^{\mathrm{g}, \mathrm{h}}$, E. Campagna $^{\mathrm{d}, \mathrm{e}}$, , B. Canuel $^{\mathrm{b}}$, F. Carbognani ${ }^{\mathrm{b}}$, F. Cavalier ${ }^{\mathrm{t}}$, R. Cavalieri ${ }^{\mathrm{b}}$, G. Cella ${ }^{\mathrm{l}}$, E. Cesarini ${ }^{\mathrm{e}}$, E. Chassande-Mottin ${ }^{\mathrm{a}}$, A. Chincarini ${ }^{\mathrm{f}}$,

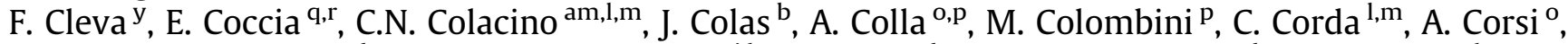
J.-P. Coulon ${ }^{\mathrm{y}}$, E. Cuoco ${ }^{\mathrm{b}}$, S. D’Antonio ${ }^{\mathrm{q}}$, A. Dari ${ }^{\mathrm{j}, \mathrm{k}}$, V. Dattilo ${ }^{\mathrm{b}}$, M. Davier ${ }^{\mathrm{t}}$, R. Day ${ }^{\mathrm{b}}$, R. De Rosa ${ }^{\mathrm{g}, \mathrm{h}}$, M. Del Prete ${ }^{1, n}$, L. Di Fiore ${ }^{g}$, A. Di Lieto ${ }^{1, m}$, M. Di Paolo Emilio ${ }^{\text {q,s }}$, A. Di Virgilio ${ }^{1}$, A. Dietz $^{\text {al,u }}{ }^{\text {, M. Drago }}{ }^{\text {ac,ad }}$, V. Fafone ${ }^{\mathrm{q}, \mathrm{r}}$, I. Ferrante ${ }^{\mathrm{l}, \mathrm{m}}$, F. Fidecaro ${ }^{\mathrm{l}, \mathrm{m}}$, I. Fiori ${ }^{\mathrm{b}}$, R. Flaminio ${ }^{\mathrm{v}}$, J.-D. Fournier ${ }^{\mathrm{y}}$, J. Franc ${ }^{\mathrm{v}}$, S. Frasca ${ }^{\mathrm{o}, \mathrm{p}}$, F. Frasconi ${ }^{1}$, A. Freise an , L. Gammaitoni ${ }^{\mathrm{j}, \mathrm{k}}$, F. Garufi ${ }^{\mathrm{g}, \mathrm{h}}$, G. Gemme $^{\mathrm{f}}$, E. Genin ${ }^{\mathrm{b}}$, A. Gennai $^{1}$, A. Giazotto ${ }^{1}$, M. Granata ${ }^{\text {a }}$, C. Greverie ${ }^{\mathrm{y}}$, G. Guidi ${ }^{\mathrm{d}, \mathrm{e}}$, H. Heitmann ${ }^{\mathrm{y}, \mathrm{z}}$, P. Hello ${ }^{\mathrm{t}}$, S. Hild ${ }^{\text {an }}$, D. Huet ${ }^{\mathrm{b}}$, P. Jaranowski ${ }^{\text {ai }}$, I. Kowalska ag , A. Królak ${ }^{\text {ae,aj, }}$ P. La Penna ${ }^{b}$, N. Leroy ${ }^{t}$, N. Letendre ${ }^{u}$, T.G.F. Li ${ }^{\text {w }}$, M. Lorenzini ${ }^{d}$, V. Loriette ${ }^{c}$, G. Losurdo $^{\mathrm{d}}$, J.-M. Mackowski ${ }^{\mathrm{v}}$, E. Majorana ${ }^{\mathrm{o}}$, N. Man ${ }^{\mathrm{y}}$, M. Mantovani ${ }^{\mathrm{l}, \mathrm{n}}$, F. Marchesoni $^{\mathrm{j}}$, F. Marion $^{\mathrm{u}}$, J. Marque $^{\mathrm{b}}$, F. Martelli ${ }^{\mathrm{d}, \mathrm{e}}$, A. Masserot $^{\mathrm{u}}$, F. Menzinger ${ }^{\mathrm{b}}$, C. Michel $^{\mathrm{v}}$, L. Milano ${ }^{\text {g,h }}$, Y. Minenkov ${ }^{\mathrm{q}}$, M. Mohan $^{\mathrm{b}}$, J. Moreau ${ }^{c}$, N. Morgado v, A. Morgia ${ }^{\mathrm{q}, \mathrm{r}}$, S. Mosca ${ }^{\mathrm{g}, \mathrm{h}}$, V. Moscatelli ${ }^{\mathrm{o}}$, B. Mours ${ }^{\mathrm{u}}$, I. Neri ${ }^{\mathrm{j}, \mathrm{k}}$, F. Nocera $^{\mathrm{b}}$, G. Pagliaroli q,r, C. Palomba ${ }^{\text {o, F. Paoletti }{ }^{\text {l,b }}, \text { S. Pardi }}{ }^{\text {g,h }}$, M. Parisi ${ }^{\text {h }}$, A. Pasqualetti ${ }^{\text {b }}$, R. Passaquieti ${ }^{1, m}$, D. Passuello ${ }^{1}$, G. Persichetti ${ }^{\text {g,h }}$, M. Pichot ${ }^{\mathrm{y}}$, F. Piergiovanni ${ }^{\mathrm{d}, \mathrm{e}}$, M. Pietka $^{\mathrm{ag}}$, L. Pinard ${ }^{\mathrm{v}}$, R. Poggiani ${ }^{\mathrm{l}, \mathrm{m}}$, M. Prato ${ }^{f}$, G.A. Prodi ${ }^{\text {aa,ab }}$, M. Punturo ${ }^{\mathrm{j}}$, P. Puppo ${ }^{\circ}$, O. Rabaste ${ }^{\mathrm{a}}$, D.S. Rabeling ${ }^{\mathrm{w}, \mathrm{x}}$, P. Rapagnani $^{\mathrm{o}, \mathrm{p}}$, V. Re $^{\mathrm{aa}, \mathrm{ab}}$, T. Regimbau ${ }^{\mathrm{y}}$, F. Ricci ${ }^{\mathrm{o}, \mathrm{p}}$, F. Robinet ${ }^{\mathrm{t}}$, A. Rocchi ${ }^{\mathrm{q}}$, L. Rolland ${ }^{\mathrm{u}}$, R. Romano $^{\mathrm{g}, \mathrm{i}}$, D. Rosińska $^{\text {ak,ah }}{ }^{\text {, P. Ruggi }}{ }^{\mathrm{b}}$, F. Salemi ${ }^{\text {aa,ab }}$, B. Sassolas v , D. Sentenac ${ }^{\text {b }}$, R. Sturani ${ }^{\text {d,e }}$, B. Swinkels ${ }^{\text {b }}$, A. Toncelli ${ }^{1, m}$, M. Tonelli ${ }^{1, m}$,

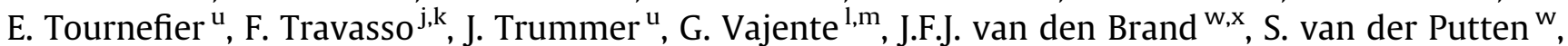

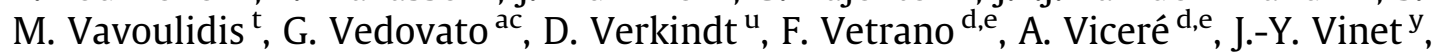
H. Vocca ${ }^{\mathrm{j}}$, M. Was ${ }^{\mathrm{t}}$, M. Yvert ${ }^{\mathrm{u}}$

\footnotetext{
${ }^{a}$ AstroParticule et Cosmologie (APC), CNRS: UMR7164-IN2P3 - Observatoire de Paris-Université Denis Diderot-Paris VII - CEA: DSM/IRFU, France

${ }^{\mathrm{b}}$ European Gravitational Observatory (EGO), I-56021 Cascina (Pisa), Italy

${ }^{\mathrm{c}}$ ESPCI, CNRS, F-75005 Paris, France

'INFN, Sezione di Firenze, I-50019 Sesto Fiorentino, Italy

e Università degli Studi di Urbino 'Carlo Bo', I-61029 Urbino, Italy

${ }^{\mathrm{f}}$ INFN, Sezione di Genova, I-16146 Genova, Italy

IINF, Sezione di Napoli, Italy

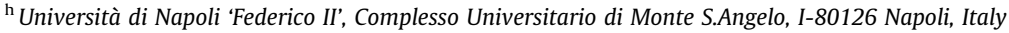

${ }^{\mathrm{i}}$ Università di Salerno, Fisciano, I-84084 Salerno, Italy

${ }^{\mathrm{j}}$ INFN, Sezione di Perugia, Italy

${ }^{\mathrm{k}}$ Università di Perugia, I-6123 Perugia, Italy

${ }^{1}$ INFN, Sezione di Pisa, Italy

${ }^{m}$ Università di Pisa, I-56127 Pisa, Italy

${ }^{\mathrm{n}}$ Università di Siena, I-53100 Siena, Italy

${ }^{\circ}$ INFN, Sezione di Roma, Italy

' Università 'La Sapienza', I-00185 Roma, Italy

${ }^{\mathrm{q}}$ INFN, Sezione di Roma Tor Vergata, Italy

${ }^{\mathrm{r}}$ INFN, Università di Roma Tor Vergata, Italy

${ }^{\mathrm{s}}$ Università dell'Aquila, I-67100 L'Aquila, Italy

${ }^{\mathrm{t}}$ LAL, Université Paris-Sud, IN2P3/CNRS, F-91898 Orsay, France

'Laboratoire d'Annecy-le-Vieux de Physique des Particules (LAPP), IN2P3/CNRS, Université de Savoie, F-74941 Annecy-le-Vieux, France

${ }^{\mathrm{v}}$ Laboratoire des Matériaux Avancés (LMA), IN2P3/CNRS, F-69622 Villeurbanne, Lyon, France
}

\footnotetext{
* Corresponding author. Tel.: +39 338 2456301; fax: +39 050752550.

E-mail address: stefano.braccini@pi.infn.it (S. Braccini).
} 


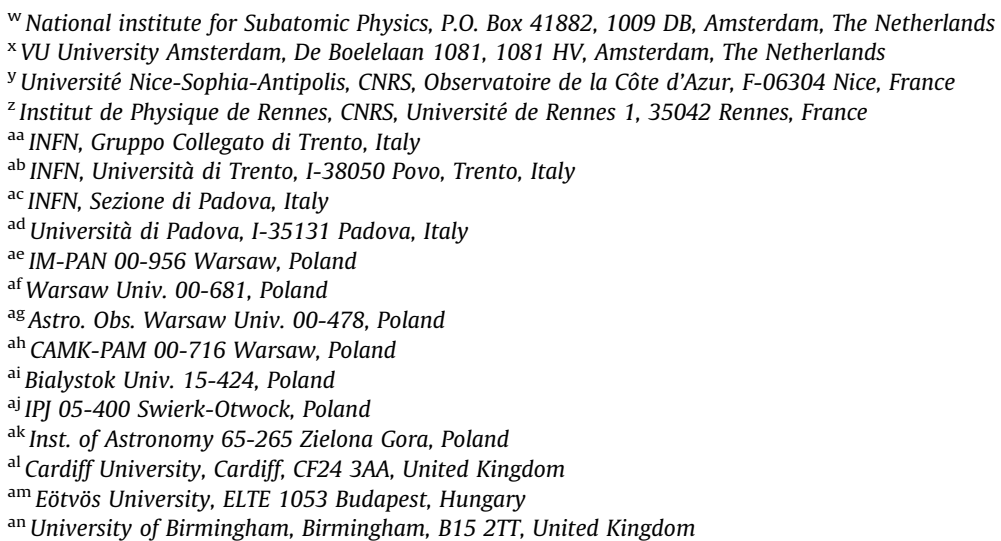

\section{A R T I C L E I N F O}

Article history:

Received 17 November 2009

Received in revised form 15 January 2010

Accepted 17 January 2010

Available online 30 January 2010

Keywords:

Gravitational wave detectors

Suspensions

Seismic isolation

\begin{abstract}
A B S T R A C T
Each mirror of the interferometric gravitational wave antenna Virgo is attached to a Superattenuator, a chain of mechanical filters designed to suppress seismic vibrations, starting from a few $\mathrm{Hz}$. The filter chain attenuation has been measured by exciting its suspension point with sinuisodal forces and using the interferometer as sensor. The attenuation, measured at different frequencies, is compliant with the requirements of the next generation antenna Advanced Virgo. In the third generation detector Einstein Telescope, the attenuation is sufficient above $3 \mathrm{~Hz}$, independently of the underground site choice.
\end{abstract}

(c) 2010 Elsevier B.V. All rights reserved.

\section{Introduction}

Virgo [1] is a suspended recycled Michelson interferometer with two orthogonal arms along which $3 \mathrm{~km}$-long Fabry-Perot cavities are accommodated. The detector is located at the European Gravitational Wave Observatory (EGO), close to Cascina (Pisa), and is designed to detect gravitational waves emitted by astrophysical sources between $10 \mathrm{~Hz}$ and a few $\mathrm{kHz}$. A gravitational wave impinging on the plane defined by the two arms of the suspended interferometer is expected to stretch one arm and to shrink the other, alternatively in each half-period. In the detection band (i.e below a few $\mathrm{kHz}$ ) the gravitational wavelength is much larger than the interferometer dimensions. In this approximation, the differential deformation of the arms $\Delta L$ can be seen as produced by a tidal force, with opposite sign on the two axes. The differential deformation $\Delta L$ results to be proportional to the arm length $L$ and to the gravitational wave dimensionless amplitude $(h(t))$, in accordance with the relationship $\Delta L(t) \simeq h(t) L$ [2]. Even considering km-long arms, the most promising signals do not induce differential arm length variations larger than $10^{-20}-10^{-18} \mathrm{~m}$. In order to detect so weak a signal the interferometer has to be characterized by an extremely high sensitivity. In the tens of $\mathrm{Hz}$ spectral region and below, ground seismic vibrations are billions of times larger than the small deformation of the arms to be detected. All around the world several systems to isolate the mirrors of various detectors from seismic noise have been developed by using both passive mechanical attenuators and active feedback techniques [3-7]. In Virgo, each mirror has been attached to a chain of mechanical filters (Superattenuator - SA) designed to filter the vibrations by more than ten orders of magnitude in all degrees of freedom, starting from a few $\mathrm{Hz}$.

The antenna sensitivity is usually expressed by the linear spectral density (i.e. the square root of the power spectrum) of the dimensionless amplitude $h$ of the equivalent gravitational signal that would produce the measured noise spectrum at the interferometer output (the photodiode where the interference takes place). This quantity has $H z^{-1 / 2}$ units. The antenna sensitivity to a differential arm displacement (typically expressed in $\mathrm{m} \cdot \mathrm{Hz}^{-1 / 2}$ units) can be obtained, according to the previous relationship $(\Delta L(t) \simeq h(t) L)$, multiplying the $h$-sensitivity curve by the arm length $L$.

The second scientific run of Virgo started on July 2009, in coincidence with two gravitational-wave interferometric antennae of the LIGO project in the US [8]. The network will increase the confidence in the detection of the extremely weak signals induced by gravitational waves. A typical Virgo sensitivity curve (obtained a few months after the run start) is plotted in Fig. 1.

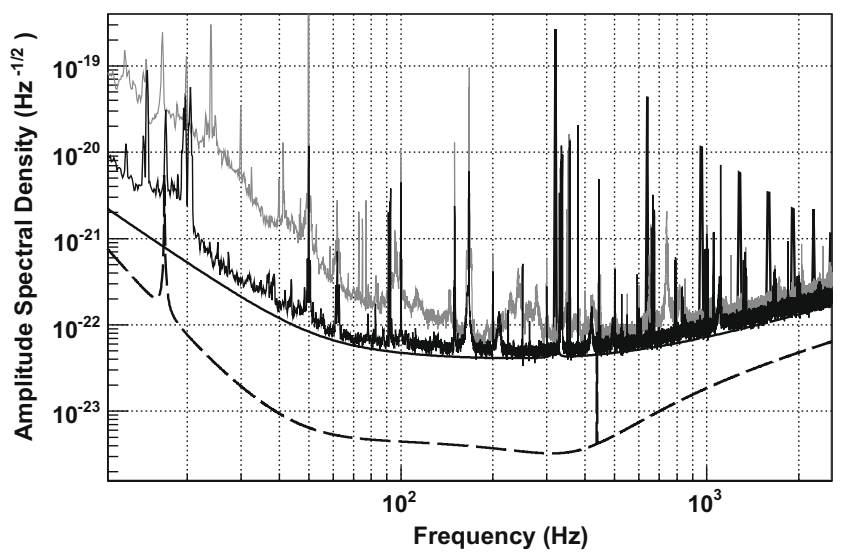

Fig. 1. Virgo sensitivity achieved after a few months of the second scientific run (VSR2 - black experimental curve), compared with the sensitivity reached in 2007 during the first scientific run (VSR1 - gray curve). The upper continuous curve is the Virgo design sensitivity, discussed in [9], while the dotted curve is the design sensitivity of Advanced Virgo, the next generation detector. The sensitivity during our tests was just slightly worse than the VSR2 one (a factor 2-3 in the tens of $\mathrm{Hz}$ region, depending on the test period) 


\section{The Superattenuator}

The SA (Fig. 2), described in [10], is essentially a five-stage pendulum supported by a three-leg elastic structure, called Inverted Pendulum [11]. In an $\mathrm{N}$-stage pendulum the horizontal displacement of the suspension point, at a frequency $f$ much higher than its normal modes $\left(f_{1}, f_{2}, \ldots, f_{N}\right)$, is transmitted to the last stage reduced by a factor proportional to $f^{2 N}$. In particular, the ratio between the linear spectral density of the last mass displacement (the optical component) and the linear spectral density of the suspension point displacement (where the excitation is applied), goes as $A / f^{2 N}$, where $A=f_{1}^{2} \cdot f_{2}^{2} \cdot \ldots \cdot f_{N}^{2}$. Designing a long pendulum chain (about $8 \mathrm{~m}$ ) all the normal modes have been confined below $2 \mathrm{~Hz}$, in order to have an attenuation of the ground horizontal seismic noise by more than 10 orders of magnitude, starting from a few $\mathrm{Hz}$. The vertical seismic vibrations are not attenuated by the pendulum chain. This would represent a serious problem. Indeed the vertical vibrations of the mirror are partially transferred to the horizontal direction of the interferometer beam by unavoidable mechanical couplings (estimated to be well below one per cent) and by the curvature of the Earth. ${ }^{1}$ For this reason, each mass of the multi-stage pendulum is replaced by a cylindrical mechanical filter [12] with a set of concentric cantilever blade springs with low stiffness. The blades support, through an about $1 \mathrm{~m}$-long steel wire, the next mechanical filter, forming a chain of low frequency oscillators, also in the vertical direction. The blades work in parallel with a magnetic anti-spring system [12], assembled on each filter, and designed to reduce its fundamental vertical frequency from about $1.5 \mathrm{~Hz}$ down below $0.5 \mathrm{~Hz}$. Thanks to the anti-springs the vertical modes of the chain are all below $2 \mathrm{~Hz}$. The mirror is thus well isolated also from the vertical seismic noise, starting from a few $\mathrm{Hz}$.

The Optical Payload [13] is suspended by a steel wire from the last filter of the chain and is composed by three elements: the Marionette, the mirror and its Reference Mass. The Reference Mass and the mirror are suspended in parallel from the Marionette, each by two pairs of thin metallic wires, forming a cradle, and with a pendulum frequency around $600 \mathrm{mHz}$. The Marionette has four arms with a permanent magnet screwed to the end of each one. Each magnet faces a coil attached at the end of four $1 \mathrm{~m}$-long-cylinders, bolted to the bottom part of the last filter of the $S A$. This coil-magnet system allows to steer the payload in three degrees of freedom (the translation along the beam and the rotations around the vertical axis and around the horizontal one perpendicular to the beam). A fine mirror position control along these degrees of freedom is obtained by using four coil-magnet pairs, with the coils screwed on the Reference Mass and the magnets glued on the reverse side of the mirror.

Between $200 \mathrm{mHz}$ and $2 \mathrm{~Hz}$, i.e. below the detection band, the seismic excitation is amplified by the normal modes of the filter chain, making the mirror swing along the beam by more than ten microns. This displacement has to be actively controlled at the level of the optical payload to maintain the interferometer in the longitudinal working position, with a high degree of accuracy. ${ }^{2}$ Nevertheless, a too large compensation force applied close to the mirror is not acceptable, because any electro-mechanical actuation system has finite dynamics. A white noise force, proportional to the maximum required compensation amplitude, affects the mirror in

\footnotetext{
1 The $3 \mathrm{~km}$-distant plumb lines are not parallel because of the curvature of the Earth, with a misalignment of about $3 \cdot 10^{-4} \mathrm{rad}$. At least one of the two suspended mirrors of the cavity has thus to be inclined with respect to the vertical plumb line to keep them parallel. This misalignment with respect to the local vertical direction implies a transmission of the mirror vertical displacement along the beam by a factor $3 \cdot 10^{-4}$.

2 An accuracy of $10^{-12}-10^{-10} \mathrm{~m}$ in the mirror positioning is necessary to keep the cavities at resonance and a good destructive interference condition at the antenna output.
}

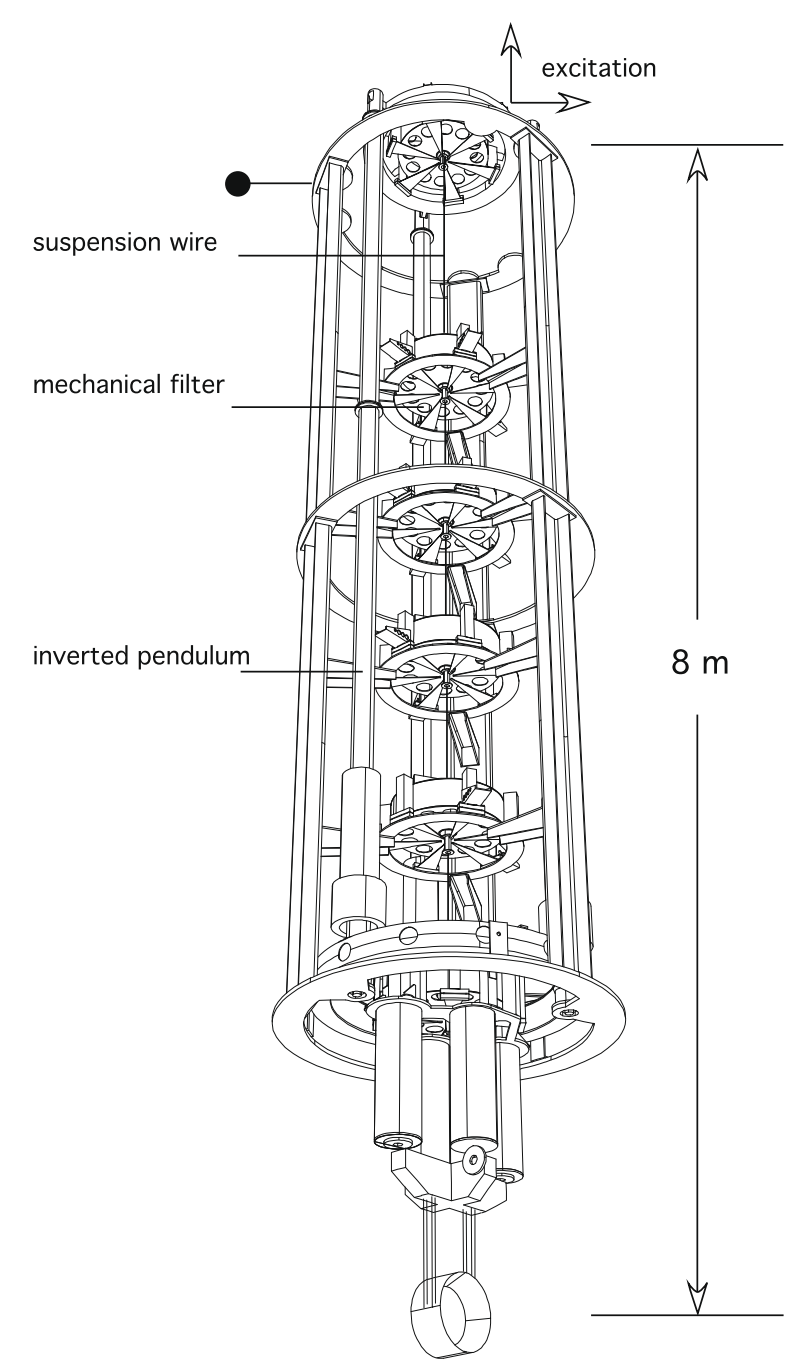

Fig. 2. The Virgo Superattenuator suppresses the transmission of ground seismic vibrations to the suspended mirror. The mechanical filter chain and the three legs of the inverted pendulum are visible. In our attenuation measurements the excitation is applied to the filter chain suspension point.

the entire band. With the present dynamics of the digital-to-analog converter (DAC board) used for the digital payload control (a noise floor of $300 \mathrm{nV} \cdot \mathrm{Hz}^{-1 / 2}$ over a range of $\pm 10 \mathrm{~V}$ [14]), the maximum adjustable horizontal displacement by using the payload actuators (without affecting the detector sensitivity) is of a few microns. A preliminary reduction of the mirror swing induced by the chain resonances is thus necessary. This is done by attaching the chain suspension point to another mechanical filter, named Filter Zero, suspended by means of three short metallic wires from the top of the Inverted Pendulum. The elasticity of the Inverted Pendulum is obtained by a steel joint through which each single leg is anchored on the bottom ring, resting on the ground. Tuning the main oscillation frequencies of the Inverted Pendulum along the two horizontal directions at about $30-40 \mathrm{mHz}$ a good horizontal preattenuation is achieved, also in the range where the chain resonances are located. An inertial control system (Inertial Damping), acting on the suspension top stage, is used to further decrease the mirror swing [15]. It is based on three high sensitivity accelerometers [16] developed for the purpose, and assembled on the Inverted Pendulum top stage in a pinwheel configuration. The accelerometers are used to monitor the suspension point acceleration in the horizontal plane (two translations and one rotation of the Filter Zero about the vertical axis) with a high accuracy. Three 
coil-magnet actuators are used in feedback, to keep the position of the top stage locked to an inertial frame. A damping of the $S A$ mechanical resonances has been obtained in this way. A similar approach is used to damp the vertical resonances. Two accelerometers and two coil-magnet actuators are used in feedback on the Filter Zero blades, at which the chain suspension point is attached.

A hierarchical control strategy is adopted to further reduce the mirror swing, and thus the control noise. The ultra-low frequency mirror displacements (below $10 \mathrm{mHz}$ ), induced by drifts and Earth tides, are very large (hundreds of microns). They are compensated using the interferometer as sensor and the three coil-magnet pairs of the horizontal Inertial Damping as feedback actuators [17]. In this case, the huge electro-mechanical floor induced by the large actuation is suppressed by the strong filtering of the entire pendulum chain underneath. The residual payload displacement along the beam (around one micron after the action of the Inertial Damping and tidal control) is compensated for from the Marionette, acting up to a few Hz. The induced electro-mechanical noise floor is suppressed down below the sensitivity curve by the mirror pendulum underneath. The Reference Mass actuators, pushing directly on the mirror, are used only to compensate for the few $\mathrm{nm}$ residual displacement of the mirror, taking place above a few Hz. The measurement of the actuation noise performed on the interferometer (to be presented in a forthcoming paper) demonstrate that even the main contribution (from the Marionette actuation point), does not affect the Virgo sensitivity, not even the sensitivity of the next generation interferometer Advanced Virgo [18].

\section{Seismic noise attenuation requirements}

As shown in Fig. 1, Virgo is operating close to its design sensitivity also in the low frequency range and is presently not limited by the mirror seismic noise passing through the $S A$. The goal of our measurements is to check if the present $S A$ attenuation is compliant with the higher sensitivity of the next generation antennae. The mechanical transfer function requirements for isolation systems in future detectors can be obtained starting from their design sensitivity curve, expressed in term of displacement (Fig. 3). The curves refer to Advanced Virgo [19], the next generation $3 \mathrm{~km}-\mathrm{long}$ interferometer expected to enter in action in 2014 at the Virgo site, and to the two reference configurations of the third generation antenna (Einstein Telescope), presently in the design study phase [20]. The goal of the $10 \mathrm{~km}$-long arm interferometer, to be located in an

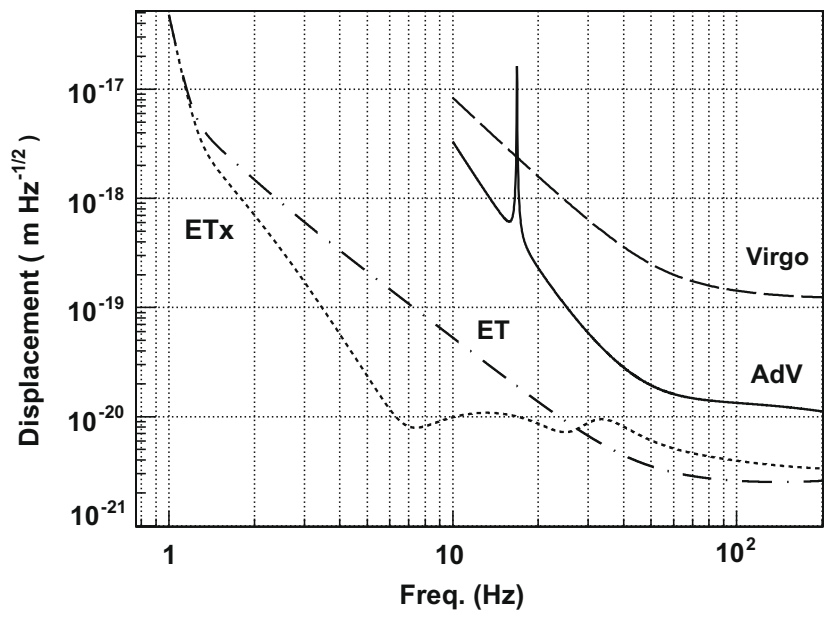

Fig. 3. Design displacement sensitivities of Virgo, Advanced Virgo (AdV), and of the two reference configurations of the third generation antenna Einstein Telescope: the standard set-up (ET) and the 'xylophone' design (ETx), optimized for the low frequency detection. While the detection band of Virgo and AdV starts from $10 \mathrm{~Hz}$, Einstein Telescope aims to reduce the low frequency threshold down to a few Hz.

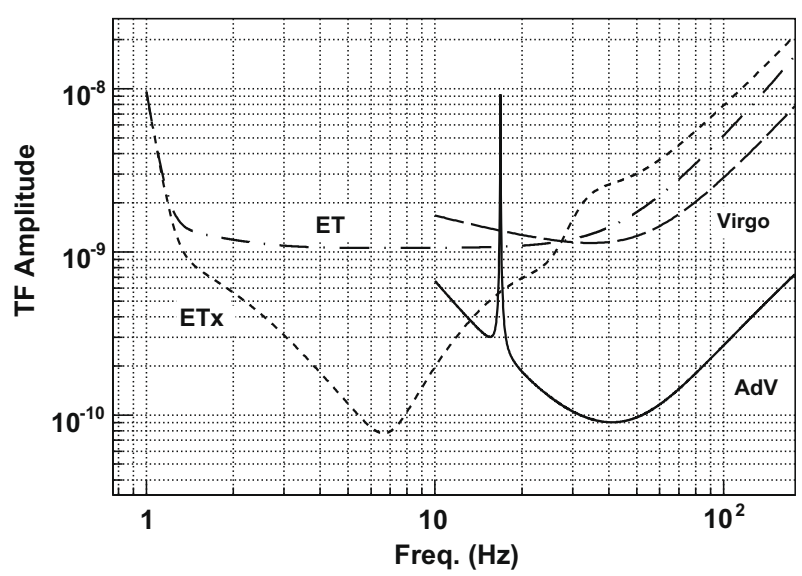

Fig. 4. Seismic vibration transfer function requirements for the different antennae. The curves are obtained dividing the displacement sensitivities displayed in Fig. 3 by the conservative linear spectral density of the seismic noise at the ground level. Since the seismic noise will be at least a couple of orders of magnitude smaller underground, Einstein Telescope, despite its better sensitivity, is less demanding in terms of seismic attenuation at high frequency.

underground site (still to be selected), is the improvement of the sensitivity achievable by second generation detectors by about a factor ten in the whole detection band (between $10 \mathrm{~Hz}$ and a few $\mathrm{kHz}$ ). An additional improvement will be the reduction of the detection band low frequency threshold from $10 \mathrm{~Hz}$ down to a few $\mathrm{Hz}$.

The maximum acceptable transfer function amplitude of the seismic isolation system in different antennae is plotted as a function of the frequency in Fig. 4. This is given by the ratio between the detector displacement sensitivity curves of Fig. 3 and the input seismic noise linear spectral density measured on the ground. At the Advanced Virgo site (the same of Virgo) the linear spectral density of the ground seismic displacement has been measured to be roughly isotropic and approximable, between a fraction of $\mathrm{Hz}$ and a few tens of $\mathrm{Hz}$, by the function $10^{-7} / \mathrm{f}^{2} \mathrm{~m} \cdot \mathrm{Hz}^{-1 / 2}$, where $f$ indicates the spectral frequency [21]. A conservative value of $5 \cdot 10^{-7} / f^{2} \mathrm{~m} \cdot \mathrm{Hz}^{-1 / 2}$ has been considered to take into account possible fluctuations in some spectral regions, and the fact that the residual seismic noise displacement affects all four mirrors of the two Fabry-Perot cavities. ${ }^{3}$ Since the Einstein Telescope site has not yet been chosen, the requirement has to be considered as provisional. The seismic noise linear spectral density of $5 \cdot 10^{-9}$ / $f^{2} \mathrm{~m} \cdot \mathrm{Hz}^{-1 / 2}$, measured in the Kamioka mine, where the new cryogenic Japanese interferometric detector is planned to be installed [22], is taken as a reference. The recent progresses of Einstein Telescope working group for site selection are promising [23]. In particular, preliminary measurements of seismic noise in underground sites in Germany, provide seismic spectra smaller by a few units or comparable with the one measured in Kamioka. This makes the chosen reference seismic floor conservative for our goals.

It is important to stress that the transfer function requirements are valid both for the vertical and horizontal seismic noise, that have a similar magnitude. While in horizontal the argument is straightforward, in the vertical case the plot represents the maximum fraction of ground vertical seismic noise that can be transferred to the mirror along the beam direction without affecting the antenna sensitivity. It is evident that the vertical to horizontal coupling factor mentioned in the previous section (in any case less than one) helps to reach the required filtering.

\footnotetext{
${ }^{3}$ At the frequencies of our interest, the residual seismic noise on different mirrors sum up incoherently. This means that four mirrors with the same displacement noise cause, in terms of linear spectral density, twice the contribution of a single one.
} 


\section{Previous superattenuator attenuation measurements}

The residual displacement at the mirror level is extremely small even if strong excitations are applied to the $S A$ top stage. Consequently, a direct measurement of the total transfer function with commercial instrumentation is impossible. The total transfer function has been extrapolated by the measurement of the transfer function of single stages [10]. In particular, the $6 \times 6$ matrices, connecting the displacements in all the degrees of freedom of each pair of consecutive filters, have been measured by using commercial accelerometers, and are combined to estimate the total attenuation. The measured isolation for vertical and horizontal seismic noise is in excellent agreement with the Lagrangian simulation and is sufficient to reduce seismic noise below the Virgo sensitivity curve, starting from about $3 \mathrm{~Hz}$. We report in Fig. 5 the total transfer function extrapolated by the stage by stage measurement technique discussed in [10]. The curve represents the total transfer function, that is the fraction of ground seismic noise (assumed to be isotropic) that is transmitted to the mirror, towards the beam direction. As shown in [10], above a few $\mathrm{Hz}$, the total transfer function is dominated by the "vertical channel", namely by the vertical ground seismic noise that is transmitted through the mechanical filter chain and is coupled to the horizontal direction at the mirror level. The lower attenuation along the vertical axis is due to the first internal mode of the blade springs of the mechanical filters (around $100 \mathrm{~Hz}$ ) that, already starting from around $10 \mathrm{~Hz}$, induce a deviation of the $1 / f^{2}$ behavior (proper of a perfect oscillator), with a depression of the filtering. One can notice the steep slope after the range of the main resonances of the chain, all located below $2 \mathrm{~Hz}$. The other resonances appearing at higher frequencies, in the region of $10 \mathrm{~Hz}$ and more, are due to the internal modes of the SA structures (blades, suspension wires, etc.) [12].

An attempt to measure directly the total attenuation of the $S A$ filter chain has been done a few years ago at the frequency of $4.1 \mathrm{~Hz}$ [24]. A sinusoidal force has been applied at this frequency along the beam direction to the chain suspension point, by injecting a current into the Inertial Damping coil-magnet actuators. The Virgo test interferometer (Central Interferometer - CITF [25]) has been used as sensor of the mirror residual displacement. Thanks to the softness of the Inverted Pendulum a large displacement of the suspension point (fraction of microns) can be induced by a not too large current in the coils (less than $1 \mathrm{~A}$ ). Despite the good sensitivity of the CITF around $4.1 \mathrm{~Hz}$ (see fifth column of Table 1 for a comparison with Virgo) and the long duration of the measure-

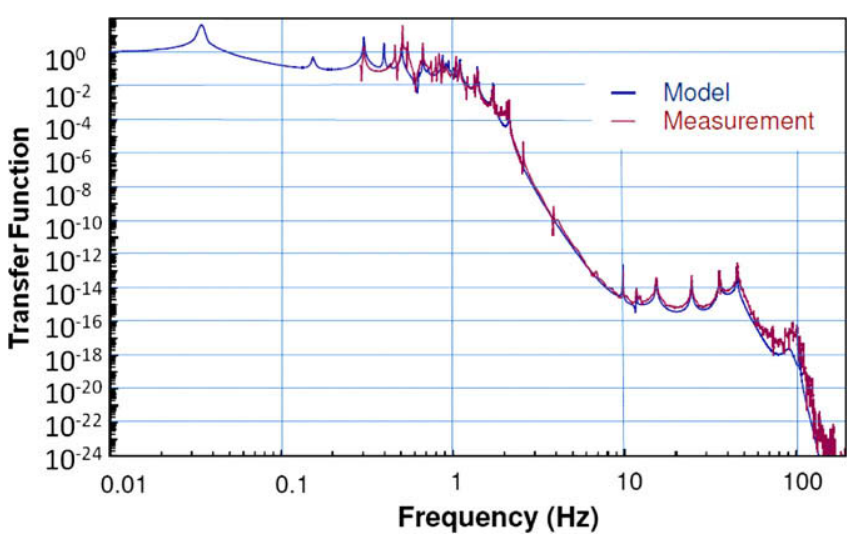

Fig. 5. The total Superattenuator transfer function extrapolated by the stage by stage measurements, using the technique described in [10]. This quantity has to be multiplied by the input seismic noise detected at the ground level, assumed to be isotropic, to obtain the residual mirror displacement along the beam.
Table 1

Excitation along the beam.

\begin{tabular}{llrllll}
\hline SA & Time $(\mathrm{s})$ & $\mathrm{f}(\mathrm{Hz})$ & $\mathrm{Top}\left(\mathrm{m} \mathrm{Hz}^{-1 / 2}\right)$ & $\operatorname{mir}\left(m \mathrm{mz}^{-1 / 2}\right)$ & TF & Note \\
\hline CITF & 10,485 & 2.25 & $4.8 \mathrm{e}-5$ & $2.5 \mathrm{e}-10$ & $5.2 \mathrm{e}-6$ & $* *$ \\
CITF & 10,485 & 4.10 & $3.3 \mathrm{e}-5$ & $2.0 \mathrm{e}-12$ & $6.1 \mathrm{e}-8$ & $<$ \\
WI & 20,971 & 4.30 & $4.3 \mathrm{e}-6$ & $3.6 \mathrm{e}-14$ & $8.4 \mathrm{e}-9$ & $<$ \\
WI & 20,971 & 6.30 & $6.0 \mathrm{e}-6$ & $5.4 \mathrm{e}-14$ & $9.0 \mathrm{e}-9$ & $<$ \\
WI & 20,971 & 10.30 & $3.0 \mathrm{e}-6$ & $1.8 \mathrm{e}-16$ & $6.0 \mathrm{e}-11$ & $<$ \\
WI & 20,971 & 18.30 & $2.8 \mathrm{e}-6$ & $4.8 \mathrm{e}-17$ & $1.7 \mathrm{e}-11$ & $<$ \\
NI & 41,943 & 29.30 & $3.0 \mathrm{e}-6$ & $1.5 \mathrm{e}-17$ & $5.1 \mathrm{e}-12$ & $* *$ \\
WI & 41,943 & 30.30 & $1.2 \mathrm{e}-6$ & $6.0 \mathrm{e}-17$ & $5.0 \mathrm{e}-11$ & $* *$ \\
NE & 20,971 & 31.30 & $9.3 \mathrm{e}-7$ & $7.5 \mathrm{e}-18$ & $8.1 \mathrm{e}-12$ & $* *$ \\
WE & 41,943 & 32.30 & $1.2 \mathrm{e}-6$ & $2.0 \mathrm{e}-18$ & $1.7 \mathrm{e}-12$ & $<$ \\
\hline
\end{tabular}

ment (hours) ${ }^{4}$, no peak at $4.1 \mathrm{~Hz}$ has been distinguished from the interferometer noise floor. As a consequence, only an upper limit of the vibration transfer function by a few $10^{-8}$ has been given for this specific frequency. This allowed concluding that already at $4.1 \mathrm{~Hz}$ (i.e. below the Virgo detection band, which starts at $10 \mathrm{~Hz}$ ) the residual mirror displacement is smaller than the one induced by the mirror thermal noise, which limits the antenna sensitivity in the low frequency range. The same conclusion (at the frequency of $4.15 \mathrm{~Hz}$ ) has been reached also for the vertical seismic noise, by applying the current to the Inertial Damping coil-magnet actuators, controlling the vertical position of the chain suspension point. The Virgo interferometer is now working very close to its low frequency design sensitivity, offering a very sensitive apparatus for this kind of tests.

\section{Attenuation measurements in the virgo interferometer}

Several sinusoidal excitations have been applied to the $S A$ top stage (both in vertical and horizontal) in the $\mathrm{Hz}$ and tens of $\mathrm{Hz}$ range. Each measurement required the interferometer locked for several hours, close to its best sensitivity. Moreover, only a single frequency excitation has been applied to the top stage in each experiment, in order to maximize the excitation at the investigated frequency without saturating the range of coil-magnet actuators and accelerometers. As a consequence the entire set of measurements required a significant amount of interferometer commissioning time. The result of a typical experiment (with the specific excitation of the top stage at $32.3 \mathrm{~Hz}$ along the beam) is reported in Fig. 6. The linear spectral density of the top stage displacement measured by accelerometers (gray curve) is compared here with the mirror displacement detected by the interferometer (black curve). One can see that the latter is dominated by the antenna noise. Since no peak is distinguished from the noise at the mirror level, the ratio between the floor and the top stage peak amplitude gives the upper limit of the transfer function of the filter chain at this frequency. While in terms of linear spectral density an incoherent floor is independent of the measurement time, the peak amplitude on the top stage scales with the square root of the duration of the measurement. This means that the upper limit of the transfer function, given by the ratio between the noise spectral floor and the top stage peak at the excitation frequency, improves (i.e. becomes smaller) when the integration time increases. The integration time of all the experiments is the second column of Tables 1 and 2. The Virgo calibration which allows to translate the photodiode signal (in $W$ ) into the differential mirror displacement (in $m$ ) has an uncertain not exceeding $10 \%$ in the entire detection band $[26,27]$. A similar error affects the calibration of the Inertial Damping accelerometers, used to measure the top stage displacement. Since the transfer function is given by the ratio between

\footnotetext{
4 The ratio between the peak amplitude and the incoherent floor scales with the square root of the integration time.
} 


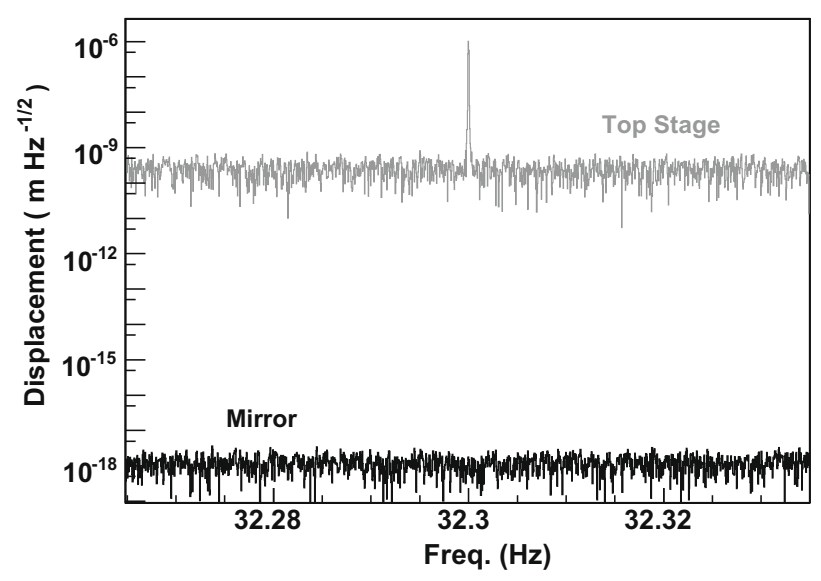

Fig. 6. The top stage of the Virgo West cavity terminal Superattenuator is shaken along the beam direction at $32.3 \mathrm{~Hz}$. The linear spectral density of top stage displacement along the beam is compared with the mirror one.

the mirror displacement detected by the interferometer and the top stage, a relative error around $20 \%$ is expected to affect the transfer function measurements. In almost all the experiments no peak has been detected at the level of the interferometer. For three cases only, around $30 \mathrm{~Hz}$, a peak at the excitation frequency has been distinguished from the interferometer noise floor (see Fig. 7). However, also in these experiments, the transfer function has been measured to not exceed a few $10^{-11}$, and is thus within the requirements displayed in Fig. 4. On the other hand, the measured transfer function for the horizontal seismic noise results to be much larger (a couple of orders of magnitude) than the one expected from the model. Facing a so accurate measurement (aimed to distinguish a residual excitation on one part on $10^{11}$ only) the possibility that other mechanisms could cause the detected thin mirror motion, by-passing the extremely high mechanical attenuation, has to be taken into account. The weak transmission between the top stage actuation coils and the mirror could be induced by spurious couplings between cables and/or electronic boards, or by antenna effects between the excitation top-stage coils and the coils used for the payload control. Even if the measurement is compliant with the attenuation requirements, the discrepancy between the model and the observed transfer function invited us to a better investigation. The experiments around $30 \mathrm{~Hz}$ have been repeated at the same frequencies, exciting the

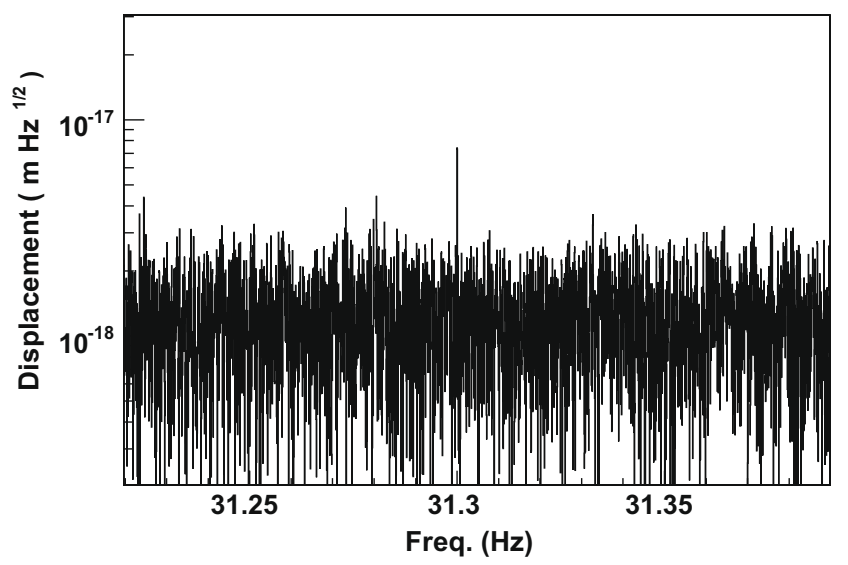

Fig. 7. With a sinusoidal $31.3 \mathrm{~Hz}$ excitation along the beam applied to the top stage of the terminal Superattenuator of the North Fabry-Perot cavity a peak is observed at the mirror level. The measurement yields a transfer function of about $8.1 \cdot 10^{-12}$ (see Table 1). top stage along the horizontal direction perpendicular to the beam. The electro-mechanical excitation amplitude, and thus the source of possible spurious effects, is similar to the previous case. Vice versa the mechanical transmission should not produce a measurable mirror displacement along the interferometer if the mechanical transfer function with the excitation along the beam has the value previously measured. In each of the three experiments, a peak has been again visible above the noise floor, with transfer functions comparable to the previous cases. Even if this is not a formal demonstration that a by-pass effect takes place, it prevents us to state that in the three cases around $30 \mathrm{~Hz}$ a direct measurement of the total $S A$ transfer function has been performed. The measurements of the vertical transfer functions made at the same frequencies, did not provide a distinguishable peak at the mirror level. It is important to stress that the vertical actuators have a completely different geometry, and that the coils are much smaller than the ones used in the horizontal case.

The upper limits achieved (and the "direct measurements") of the transfer function are reported in Fig. 8 and compared with the requirements of Fig. 4. The transfer function of the filter chain for the investigated frequencies above $10 \mathrm{~Hz}$ is below the requirement curve for all the planned future detectors. The measured upper limits below $10 \mathrm{~Hz}$ are not sufficient to state that at the investigated frequencies the filter chain alone is enough to suppress the seismic noise below the Einstein Telescope sensitivity. It is important to stress that in this region only upper limits have been set and that the expected transfer function will be likely much smaller, in agreement with the indirect stage by stage measurement reported in Fig. 5. Moreover, the few tens of $d B$ distance between the measured upper limits around 4 and $6 \mathrm{~Hz}$ and the transfer function requirement curves (reducible by longer measurements) will be covered by the antenna pre-isolator stages. Indeed, we have to stress that the extra-attenuation by pre-isolator stages is not included in our measurement, which concerns only the transfer function from the filter chain suspension point and the mirror. In Virgo, the attenuation of the Inverted Pendulum is $30-40 \mathrm{~dB}$ at $4 \mathrm{~Hz}$.

As shown in Fig. 5, going down from $4 \mathrm{~Hz}$, towards the resonance range, the transfer function exhibits a steep increase. The only direct measurement in the ultra-low frequency range has been performed on the Central Interferometer at $2.25 \mathrm{~Hz}$ both in vertical and in horizontal. The filter chain transfer function has

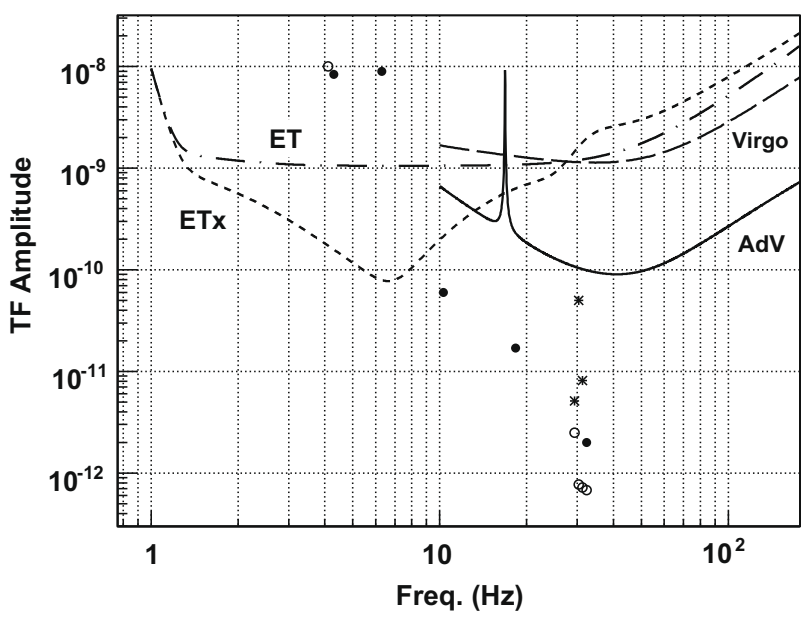

Fig. 8. The transfer functions measured in the various experiments (see Tables 1 and 2) compared to the requirements of Fig. 4 . The circles indicate the upper limits (full circles: excitations along the beam, open circles: vertical excitations). The three stars denote the "direct measurements", when a peak has been detected at the mirror level (with the excitation of the top stage along the beam direction). As discussed in the text, the relative error on the point position is around $20 \%$. 
Table 2

Vertical Excitation.

\begin{tabular}{llrllll}
\hline SA & Time $(\mathrm{s})$ & $f(\mathrm{~Hz})$ & $\begin{array}{l}\text { Top } \\
(\mathrm{m} \mathrm{Hz}-1 / 2\end{array}$ & $\begin{array}{l}\text { mir } \\
\left(\mathrm{m} \mathrm{Hz}^{-1 / 2}\right)\end{array}$ & TF & Note \\
\hline CITF & 2620 & 2.25 & $1.7 \mathrm{e}-4$ & $2.6 \mathrm{e}-10$ & $1.5 \mathrm{e}-6$ & $* *$ \\
CITF & 2620 & 4.10 & $3.0 \mathrm{e}-4$ & $3.0 \mathrm{e}-12$ & $1.0 \mathrm{e}-8$ & $<$ \\
NI & 25,165 & 29.30 & $1.2 \mathrm{e}-6$ & $3.0 \mathrm{e}-18$ & $2.5 \mathrm{e}-12$ & $<$ \\
WI & 25,165 & 30.30 & $3.9 \mathrm{e}-6$ & $3.0 \mathrm{e}-18$ & $7.7 \mathrm{e}-13$ & $<$ \\
NE & 25,165 & 31.30 & $4.1 \mathrm{e}-6$ & $3.0 \mathrm{e}-18$ & $7.2 \mathrm{e}-13$ & $<$ \\
WE & 25,165 & 32.30 & $4.4 \mathrm{e}-6$ & $3.0 \mathrm{e}-18$ & $6.8 \mathrm{e}-13$ & $<$
\end{tabular}

Results of the measurements - Column (a) Superattenuator under test (CITF refers to the old measurements on the Virgo Central Interferometer [24], 'W' and 'N' indicate the cavity of the Superattenuator under test, West or North, while 'I' and 'E' its position in the cavity, Input or End); Column (b) Measurement duration; Column (c) Excitation frequency; Column (d) Peak value of the linear spectral density of top stage displacement at the excitation frequency, measured by top stage accelerometers; Column (e) Linear spectral density of the mirror displacement along the beam at the excitation frequency, as detected by the interferometer; Column (f) Transfer function amplitude (ratio between the previous two columns); Column ( $\mathrm{g}$ ) Measurement outcome (the symbol " $<$ " denotes that only an upper limit can be given, while the two stars indicate that a peak has been detected at the mirror level). As mentioned above, the accuracy of the transfer function measurements, dominated by the calibration errors, is around $20 \%$.

been measured to be a few parts per million in both cases (Tables 1 and 2). The gap with the requirements in the $2 \mathrm{~Hz}$ region is thus very large and can be covered only by conceiving high performance pre-isolator stages. One can thus conclude that at $2-2.5 \mathrm{~Hz}$ the present $S A$ is likely not compliant with Einstein Telescope. Looking at the steep behavior of the indirect transfer function measurement (Fig. 5) one can infer that, assuming the use of the present $\mathrm{SA}$, the crossing between the mirror residual seismic noise and the Einstein Telescope sensitivity should take place around $3 \mathrm{~Hz}$. Since the transfer function changes by many orders of magnitude in a few tenths of $\mathrm{Hz}$, this conclusion is independent of the selection of the Einstein Telescope site. Even a large change of ground seismic noise (and consequently of the isolation specifications) does not affect macroscopically the crossing frequency between the steep transfer function and the attenuation requirement curve. A crossing around $3 \mathrm{~Hz}$ will take place even shifting by two orders of magnitude the reference curves in Fig. 4 along the vertical axis. The Einstein Telescope low frequency detection threshold is still not fixed, since the present Newtonian noise estimates are high, making difficult a good sensitive detection below a few $\mathrm{Hz}$. This noise mechanism, described in [28], is induced by the gravitational coupling of the mirror with the ground mass distribution, varying because of seismic waves.

\section{Conclusions}

The suspension point of the Virgo Superattenuator filter chain has been excited several times both in horizontal and in vertical directions, injecting sinusoidal signals at different frequencies into the top stage coil-magnet actuators. In almost all the measurements, the mirror residual motion at the excitation frequency is too small to be distinguished from the interferometer noise floor, and only upper limits for the transfer function have been set. In three tests, around $30 \mathrm{~Hz}$, with the suspension point excitation along the beam, a peak above the interferometer floor has been detected, putting in evidence a noise transmission. There are indications that the small residual signal could be induced by other spurious mechanisms, by-passing the strong Superattenuator mechanical attenuation. In any case, at all investigated frequencies, the transfer function of the filter chain (measured directly or by upper limits) is within the requirements of Advanced Virgo, whose detection band starts from $10 \mathrm{~Hz}$. Above this frequency, the measured attenuations are compliant also with the requirements of the third generation detector Einstein Telescope that is, at frequency above $10-15 \mathrm{~Hz}$, less demanding in terms of attenuation, because the seismic noise is much lower underground.

The upper limits of the filter chain transfer function measured at lower frequencies, around 4 and $6 \mathrm{~Hz}$, are larger than the required values. The upper limit can be reduced by longer and more sensitive measurements. In addition, the gap between the present upper limits and the requirements is in any case small enough (a few tens of $\mathrm{dB}$ ) to be covered by the additional attenuation coming from the pre-isolator stages, not included in our measurement. This argument, together with the indirect measurement of attenuation valid at all frequencies, allows concluding that modifications to the Superattenuator (such as a lengthening of the chain) will be necessary in Einstein Telescope only if the detection threshold frequency will be moved below $3 \mathrm{~Hz}$. One can notice that the transfer function is very steep around $3 \mathrm{~Hz}$. As a consequence, even changing by a couple of orders of magnitude the input seismic noise, and thus the values of the necessary attenuation, the crossing frequency between the transfer function and the requirement curve of ET will remain around $3 \mathrm{~Hz}$.

\section{Acknowledgments}

The authors acknowledge the support of the Italian Istituto Nazionale di Fisica Nucleare and the French Centre National de la Recherche Scientique for the construction and operation of the Virgo detector. In addition, the authors acknowledge the support of the research by the Foundation for Fundamental Research on Matter of the Netherlands Organization for Scientific Research, the Polish Ministry of Science and Higher Education Grant N N203 387237, the FOCUS Programme of Foundation for Polish Science and the European Associated Laboratory Astrophysics PolandFrance. This work, part of the Einstein Telescope Design Study project supported by the European Community, has received funds from EU Seventh Framework Program FP7, under the grant agreement n.211743. The work has been carried on also thanks to the support coming from Italian Ministero dell'Istruzione, dell'Universita' e della Ricerca through the Grant PRIN 2007: "Sensibilita' a bassa frequenza in interferometri per onde gravitazionali sotterranei e criogenici".

\section{References}

[1] The Virgo collaboration, Virgo Final Design, VIR-TRE-DIR-1000-13, 1997 (available at http://pub3.ego-gw.it/itf/tds).

[2] C.W. Misner, J.A. Wheeler, K.S. Thorne, Gravitation, W.H. Freeman (Eds.), Physics Series, 1973.

[3] E.J. Chin et al., Journal of Physics, Conference Series 32 (2006) 111-116.

[4] R. Abbott et al., Class. Quant. Grav. 19 (7) (2002) 1591-1597.

[5] R. Abbott et al., Class. Quant. Grav. 21 (5) (2004) S915-S921.

[6] M.V. Plissi et al., Rev. Sci. Instrum. 71 (6) (2000) 2539-2545.

[7] A. Takamori et al., Class. Quant. Grav. 19 (7) (2002) 1615-1621.

[8] B. Abbott et al., LIGO Scientific Community, Nucl. Instr. and Meth. A 517 (2004) 154-179.

[9] M. Punturo, The Virgo sensitivity curve, Virgo Technical Report, VIR-NOT-PER1390-51, 2004 (available at http://pub3.ego-gw.it/itf/tds)

[10] G. Ballardin et al., Rev. Sci. Instrum. 72 (9) (2001) 3643-3652.

[11] G. Losurdo et al., Rev. Sci. Instrum. 70 (5) (1999) 2507-2515.

[12] M. Beccaria et al., Nucl. Instr. and Meth. A 394 (1997) 397-408.

[13] A. Bernardini et al., Rev. Sci. Instrum. 70 (8) (1999) 3463-3472.

[14] A. Gennai, Advanced Virgo - Suspension Control System, Virgo Technical Report, VIR-0120B-08, 2009 (available at http://pub3.ego-gw.it/itf/tds).

[15] G. Losurdo et al., Rev. Sci. Instrum. 72 (9) (2001) 3653-3661.

[16] S. Braccini et al., Rev. Sci. Instrum. 66 (3) (1995) 2672-2676.

[17] F. Acernese et al., Astrop. Phys. 20 (6) (2004) 629-640.

[18] Virgo Suspension group, SAT Advanced Plan, Virgo Technical Report, VIR0091A-08, 2008 (available at http://pub3.ego-gw.it/itf/tds)

[19] The Virgo collaboration, Advanced Virgo Baseline Design, Virgo Technical Report, VIR-0027A-09, 2009 (available at http://pub3.ego-gw.it/itf/tds).

[20] M. Punturo et al., Third generation gravitational wave observatories and their science reach, Proceeding of the 8th Amaldi conference on gravitational waves (June 22-26, 2009), Columbia University, New York, Class. Quant. Grav., in press. Article ID CQG/337732/SPE. (See also http://www.et-gw.eu/). 
21] F. Acernese et al., Class. Quant. Grav. 21 (5) (2004) S433-S440.

[22] M. Ohashi and LISM collaboration, Laser Interferometer in the Kamioka mine, in Proceedings of the 28th International Cosmic Ray Conference (July 31August 7, 2003), Tsukuba, Japan, Universal Academy Press, 2003.

[23] M.G. Beker et al., Einstein Telescope Working Group 1 Report - Selection criteria for ET candidate sites, ET Scientific Note, ET-030-09, 2009 (available at http://www.et-gw.eu/).

[24] S. Braccini et al., Astrop. Phys. 23 (6) (2005) 557-565.
[25] F. Acernese et al., Astrop. Phys. 21 (1) (2004) 1-22.

[26] O. Veziant et al., Class. Quant. Grav. 20 (17) (2003) S711-S720.

[27] L. Rolland, on behalf of the Virgo collaboration, "Virgo calibration and reconstruction of the gravitational wave strain during VSR1, to be published in Proceedings of the 8th Amaldi conference on gravitational waves (June 2226, 2009), Columbia University, New York.

[28] M. Beccaria et al., Class. Quant. Grav. 15 (11) (1998) 3339-3362. 